\title{
Deep subsurface bacterial proteins bind and modify clathrate
}

\author{
ABIGAIL M. JOHNSON ${ }^{1,2}$, DUSTIN J.E. HUARD ${ }^{3}$, \\ JONGCHAN KIM ${ }^{4}$, PRIYAM RAUT ${ }^{5}$, ANTON S. PETROV ${ }^{5}$, \\ LOREN D. WILlIAMS ${ }^{3}$, SHENG DAI ${ }^{4}$, RAQUEL L. \\ LIEBERMAN $^{3}$, JENNIFER B. GLASS $^{2}$ \\ ${ }^{1}$ Ocean Science \& Engineering Graduate Program, ${ }^{2}$ School of \\ Earth \& Atmospheric Sciences, ${ }^{3}$ School of Chemistry \& \\ Biochemistry, ${ }^{4}$ School of Civil \& Environmental \\ Engineering, ${ }^{5}$ School of Biology, Georgia Institute of \\ Technology, Atlanta, GA, USA \\ email: jennifer.glass@eas.gatech.edu
}

Microbial communities occur in gas clathrates on continental shelves and in permafrost. Because gas clathrates clog natural gas pipelines, companies often try to inhibit their formation using clathrate growth inhibitors, typically synthetic chemicals. Biodegradable hydrate inhibitors, including antifreeze proteins (AFPs), have similar activity. The winter-flounder AFP (wfAFP) is a particularly effective hydrate inhibitor. We hypothesized that microbial proteins in clathratecontaining marine sediments may also influence clathrate growth. We recombinantly expressed bacterial putative clathrate binding proteins (CBPs) with and without enhanced green fluorescent protein (eGFP) tags. Sequences were selected from metagenomes from methane clathrate-containing sediments from coastal Oregon and Japan based on the presence of similar amino acid motifs as wfAFP. We tested the effect of CBPs on the morphology of tetrahydrofuran (THF) clathrate and visualized THF-clathrate binding by fluorescence of eGFP tags. The positive control was wfAFP, which formed numerous platy THF crystals. Negative controls (phosphate-buffered saline solution, cytochrome, and eGFP) formed single THF crystals. We found that THF-clathrate changed morphology in the presence of CBPs: two CBPs caused the growth of small, platy crystals, similar to wfAFP, while the other three CBPs formed larger, flat crystals. eGFP-tagged CBPs showed clear evidence of clathrate binding compared to eGFP alone. Our ongoing work will establish how bacterial CBPs affect the properties of methane clathrate. It is possible that microbes use CBPs as to survive life encased in clathrate. This work is relevant for the habitability of Mars and other planetary bodies containing gas clathrates. This work was funded by a grant from the NASA Exobiology Program. 